\title{
State of knowledge and management of Ibero- american coastal aquifers with different geo-hydrological settings
}

\author{
${ }^{1}$ Federal University of Rio de Janeiro (UFRJ), Brazil. E-mail: gerson@acd.ufrj.br \\ ${ }^{2}$ Nacional University of Mar del Plata (UNMP), Argentina. E-mail: ebocaneg@mdp.edu.ar \\ ${ }^{3}$ Technical University of Catalonia (UPC), Spain. E-mail: emilio.custodio@upc.edu \\ ${ }^{4}$ Technical University of Cartagena (UPCT), Spain. E-mail: marisol.manzano@upct.es \\ ${ }^{5}$ Federal University of Pernambuco (UFP), Brazil.E-mail: suzanam@ufpe.br
}

Coastal aquifers have played a major role in modern human occupation and economic development in Iberoamerica. Most of Iberoamerica population inhabits coastal areas, and water needs are frequently fulfilled with groundwater. Possible climate changes and consequent sea level rise will deeply affect coastal aquifers, putting considerable pressure in those water bodies. For the prediction of how these aquifers will behave in face of such challenges, and also to establish preventive measures against undesired effects, adequate knowledge is essential. This paper presents the state of knowledge of a number of Latin American coastal aquifers reasonably representative of the overall situation through a literature review, with compilation and synthesis of data. A synthetic comparative analysis conducted on 33 coastal aquifers in Latin America and the Iberian Peninsula is showed, as well as detailed information about three out of those aquifers with relatively more abundant data and of particular interest: Mar del Plata, in Argentina; Recife, in Brazil; and Low Llobregat, in Spain. Some of the conclusions of the comparative analysis are: very frequently, intensive groundwater abstraction has produced marine intrusion; management actions with different technical complexity have been undertaken in some areas, although the most common situation is a lack of management at all. However, legal and regulatory frameworks to protect groundwater are slowly improving or uprising. Major challenges are unawareness on aquifer problems and underfunding for management and investigation.

\section{Introduction}

Coastal areas are transition spaces between the marine and continental domains where natural or anthropogenic processes of consumption, production and exchange of mass and energy occur at high intensity rates. Such processes exercise an intense pressure on natural resources. One of the main resources in coastal areas is groundwater, which plays a very important role as the main - and in many cases a unique - source of fresh water for human populations, and the industrial and agricultural sectors, as well as for local ecosystems. Consequently, sustainable use of groundwater resources is imperative in these locations. Creation of effective public policies towards sustainability is necessarily based on the knowledge of aquifers functioning and on the evaluation of their environmental role, hydrogeological characteristics and vulnerability and developability as a freshwater source, as well as the effective involvement of groundwater stakeholders in monitoring and management.

Sea level is expected to rise 0.6 to $1.2 \mathrm{~m}$ by the end of this century. This will increase seawater intrusion into coastal areas but with highly variable results depending on aquifer characteristics and coastal conditions, from almost imperceptible to large penetrations. This, jointly with possible recharge changes due to climatic and global factors, may induce increased risks in the areas here considered.

In order to establish preventive measures against undesired effects, adequate knowledge is essential. Thus, to predict how these changes will affect the future role of coastal aquifers and to prevent unwanted effects, when possible, it is necessary to gather and systematize available data.

This paper presents a literature review and the state of knowledge and management of a number of Iberoamerican coastal aquifers reasonably representative of the overall situation, and illustrates the work of compilation and synthesis with details of three aquifers seemingly better known: Mar del Plata, in Argentina; Recife, in Brazil; and Llobregat Delta, in Spain.

To achieve those goals, published and unpublished information on 33 coastal aquifers of the Iberian Peninsula and islands (Spain and Portugal), the Caribbean Sea (Cuba) and the Atlantic and Pacific 
coasts of Latin America (in Argentina, Brazil, Colombia, Costa Rica, Chile, Mexico, Nicaragua, Peru and Uruguay) has been extracted, synthesized and analysed (Fig.1). It is not an objective of this work to propose a classification for aquifers, but in order to manage all the information obtained as to compare and get common statements, it was helpful to group the aquifers. Taking into account the most common situations in Iberoamerican coastal areas, aquifers were divided in five operative categories: carbonated aquifers, thick detrital aquifers, small aquifers of local interest, urban and peri-urban aquifers and aquifers in small islands.

In the majority of the cases, intensive groundwater abstraction to satisfy local populations' needs or tourist-focused developments has produced significant depression of piezometric levels and marine intrusion. Additional problems include salinization caused by upconing, irrigation return flows and groundwater contamination due to lack of sewage systems and urban waste leaching in disposal areas. In some cases, the decrease of groundwater flow to streams, lagoons and wetland areas is already noticeable. Therefore, a great variety of situations such as geological settings, people's educational and income conditions, exploitation and contamination pressures, and other factors, occur in the vast area here considered.

Mar del Plata, Recife and Llobregat Delta aquifers have in common the fact that they are intensively exploited, with salinization and other pollution problems, and of great social and economic value in their settings. They also have monitoring and management plans, with public policies protecting their use and development (Bocanegra et al., 2008; Niñerola et al., 2009; Montenegro et al., 2008), so they are analysed to provide deeper insights of the studied aquifers.

The purpose of the article is therefore to present the state of knowledge about those aquifers. This will help to anticipate future developments and to assess the possible impacts of future global and climate change scenarios. Thus, in the following pages an attempt is made to look for common grounds and systematize the multitude of scenarios to portray a picture of the state of knowledge and awareness on coastal aquifers in Iberoamerica.

\section{Materials and Methods}

Scientific work on the 33 studied aquifers responds for most of the references cited here. Data from a variety of sources were compiled, integrated and typified according to Bocanegra et al. (2010) for operative purposes.

The 33 aquifers were, on a broad perspective, classified in 5 different types according to some specific characteristics: (1) Coastal aquifers in small islands are very sensitive and vulnerable. Intensive groundwater exploitation, mostly for tourist supply, has recently developed or is currently under development; (2) Carbonated coastal aquifers are prone to increasing permeability due to enhanced calcite dissolution in the mixing zone. Although this is a slow process, it has to be considered when the fresh-saltwater position is disrupted by pumping; (3) Thick detrital coastal aquifers, which are usually highly heterogeneous and anisotropic; (4) Mixed coastal aquifers, which do not show specific conditions, but may experience problems of different aquifer types simultaneously; finally, (5) Small coastal aquifers of local interest that share many features with urban and peri-urban aquifers, but their dimensions provide some specific factors.

The aquifers subjected to the extend analysis were selected due to the following factors: relative abundance and quality of data; management actions currently implemented or under planning; and being a major field work area of the authors. This lead to the choice of three aquifers: Mar del Plata, Recife and Llobregat Delta.

\section{Review of Iberoamerican Coastal Aquifers: A Summary}

\section{Main features of the studied coastal aquifers and related problems}

Coastal aquifers are bounded by the sea, a base level characterized by a local mean value that not always coincides with the topographic datum. In detail, local sea level is subject to tides, waves and wind effects, occasional rare events, marine currents and evaporation in half-closed sea water bodies. Seawater is denser than freshwater, 1.025 versus $1.000 \mathrm{~g} / \mathrm{L}$ for typical salinities and temperatures. Provided hydrodynamic forces are not significant in the groundwater body, freshwater floats on saltwater. Freshwater flow towards the coast reduces saltwater penetration into the continent, which forms a wedge that thins out landwards. The transition from freshwater to saline water may be quite sharp, thus forming an interface, or may be a more or less thick mixing zone, depending on local circumstances, the relative intensity of freshwater flow, and the fluctuations of sea and groundwater levels. Mixed (brackish) water in the ground is towed towards the coast by freshwater movement, which means that there is a marine water counterflow to compensate the salinity balance. This entire means that hydrodynamics of the continental-marine boundary is complex and depends on local circumstances of permeability, depth and layout of (heterogeneous) materials, as well as on freshwater and seawater flow oscillations (Custodio and Bruggeman, 1987).

Trends in seawater level, in the past or foreseen as a consequence of global change, modify the freshwater-saltwater relationship. In large aquifer systems, it evolves slowly, with lags of centuries to millennia. The aquitards respond especially slowly to the changes, as well as aquifers separated from the sea by aquitards. This means that taking into account water salinity distribution, the number of different coastal aquifers types is very large, and in reality each coastal aquifer, or sector of it, is unique and needs specific studies to address sustainability and management.

However, the main features of problems, management actions and needs are rather common to any type of coastal aquifer, and relevant differences are mostly related to local climate, to the country development level and to the set of available freshwater sources to supply demand. The main common features in the 33 studied coastal aquifers are:

- Groundwater salinization by different causes (induced marine intrusion; saline water upconing; connate marine water mobilization; recharge under arid conditions; infiltration of irrigation excess water). When groundwater development takes place, aquifer hydrodynamics are changed and so, consequently, the freshwater-saltwater relationships. In the case of intensive development, groundwater flow pattern may be deeply modified. This depends not only on well abstraction and penetration, but also on vertical permeability and interlayered heterogeneities. Trying to define common scenarios the following situations have to be taken into account:

- Water-table coastal aquifers, open directly to the sea. Thickness 
along the coast is important (thin and thick aquifers), jointly with permeability (large, as in karstified formations, young volcanics and well-sorted alluvium; small in other materials) and groundwater flow per unit coastal length (large if groundwater does not overflow before attaining the coast; otherwise small).

- Confined and semi-confined coastal aquifers. They can be open to the sea at an offshore position (the greater marine water density may hinder freshwater discharge into the sea bottom if freshwater head is not high enough), or separated by an aquitard or by recent sea-bottom low permeability sediments (caprock).

- More or less homogeneous formations along the shore, as in some sandy formations.

- Conspicuous heterogeneities along the coast, affecting transmissivity and aquifer bottom depth. Such are filled up incised palaeovalleys, coastal terraces and alluvial fans, main fractured zones in hard rocks and volcanics, and preferential karstic developments.

- Generalized pollution by urban, industrial and agricultural activities. In urban and peri-urban areas, where large industrial areas usually settle, the lack of sewage systems, the deficient conservation of networks or their late construction may be the cause of point but widespread groundwater pollution. Particularly in developing countries, groundwater pollution is usually observed at a rather late time, and only when generalized, serious water quality problems appear. Intensive agricultural activities are a frequent cause of coastal groundwater impairment in many areas.
- Lack of planning for groundwater management. Among other undesirable consequences, strong spatial concentrations of abstraction wells is a common feature. This leads to localized, large drawdowns that disturb the former saline-fresh water equilibrium and induces brackish and saline water to progress landward and saline water upconing, among other undesirable effects.

- Lack of monitoring networks. The existence of adequate groundwater monitoring networks in coastal aquifers is more rare than common, even in developed countries.

- Insufficient knowledge about the aquifer functioning, the existing problems, the needs for conservation, and the extent of possible anthropic impacts. This is often compound with inadequate conceptual models and lack of awareness about the risks facing groundwater resources.

\section{Typification of the studied aquifers}

Typification usefulness is to find some common feature, in order to help in the specific studies. Some specific characteristics can be drawn for the aquifer types defined with basis on the present research. They are summarized in Table 1 according to this applied classification.

For example, coastal aquifers in very small islands make any possible problem to reach easily the aquifer boundaries. Also, in many small islands intensive groundwater exploitation, mostly for tourist supply, has developed very recently or is currently under development. In all the studied aquifers of this kind, this has always happened

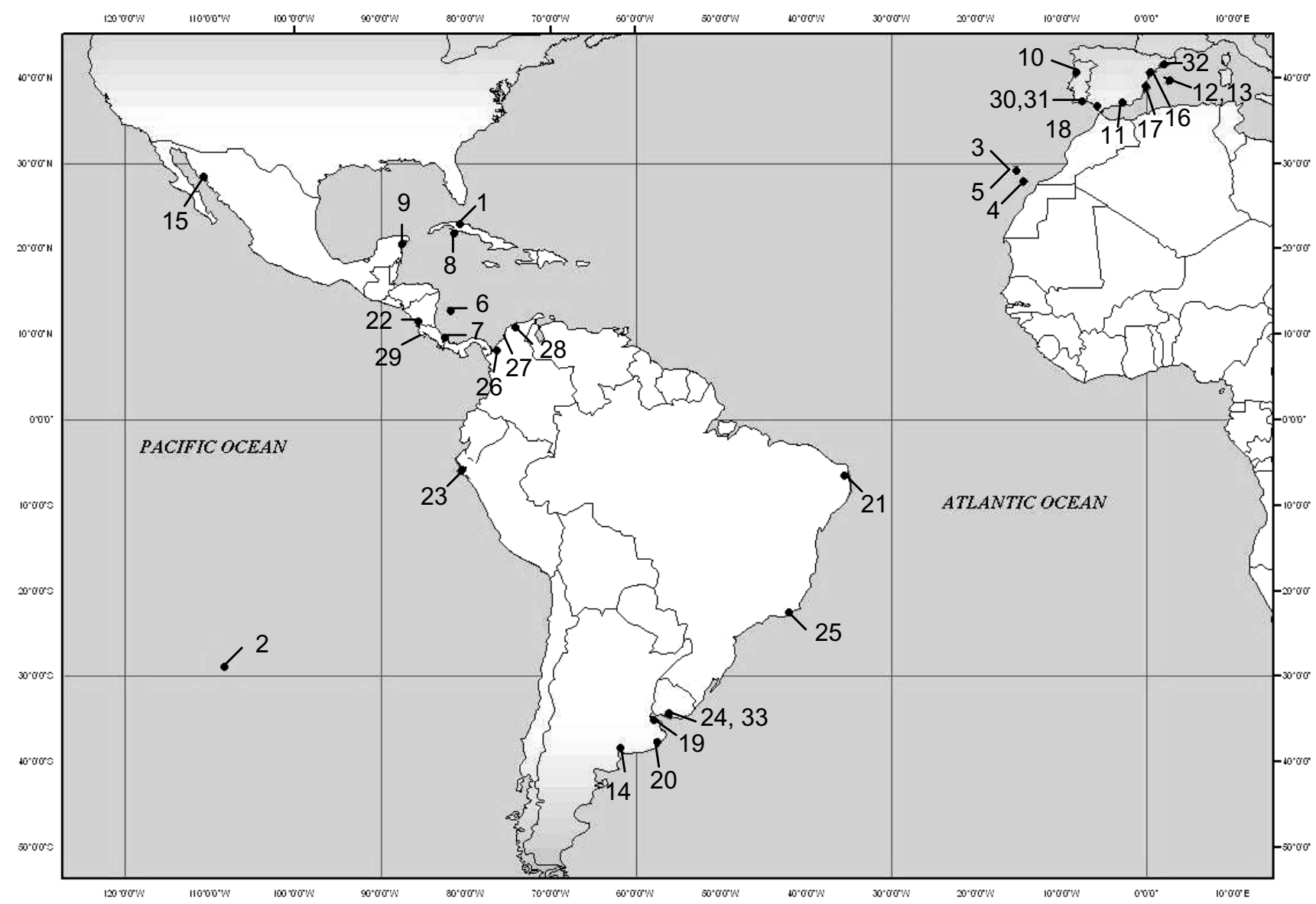

Fig. 1. Location of the studied aquifers. Numbers correspond to those in the first column of Table 1. 


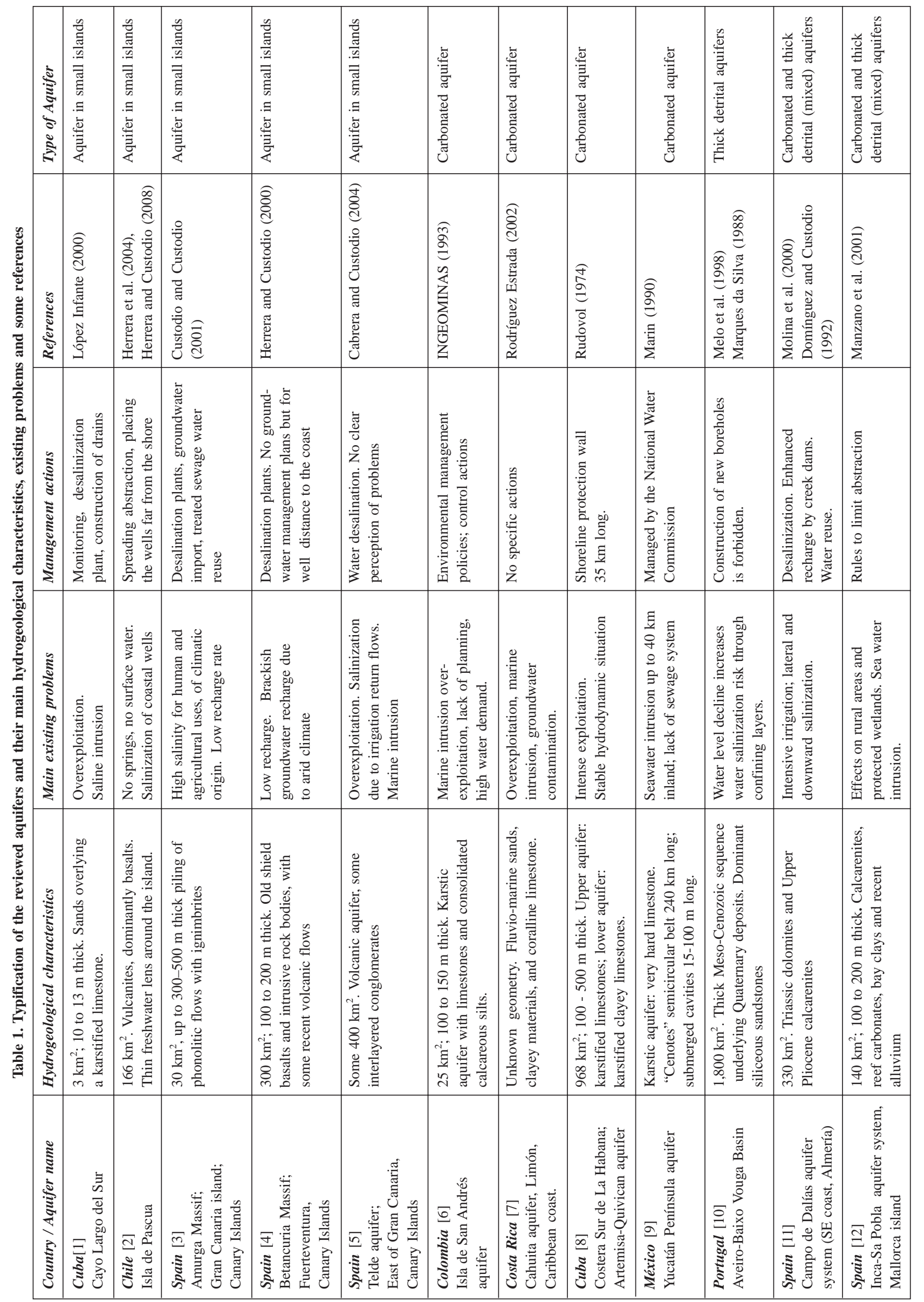




\begin{tabular}{|c|c|c|c|c|c|c|c|c|c|c|}
\hline 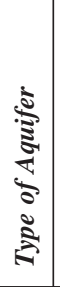 & 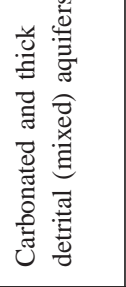 & 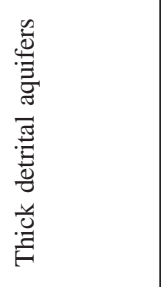 & 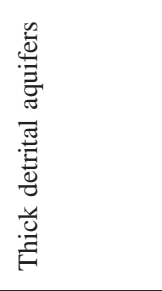 & 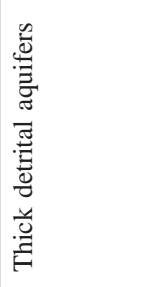 & 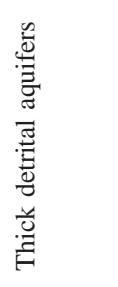 & 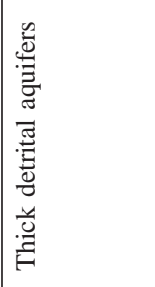 & 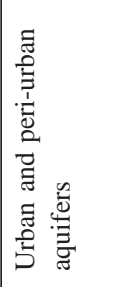 & 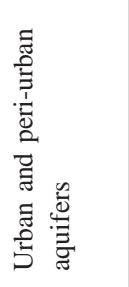 & 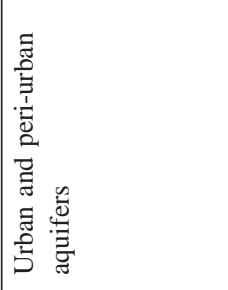 & 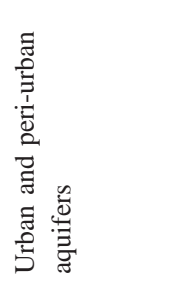 \\
\hline 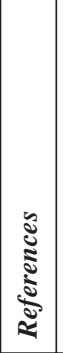 & 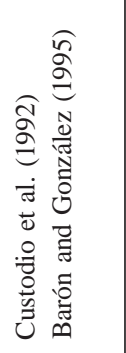 & 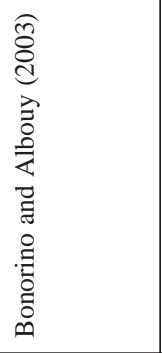 & 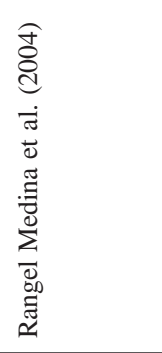 & 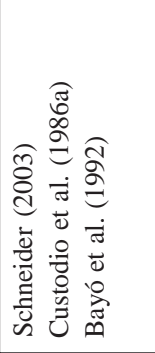 & 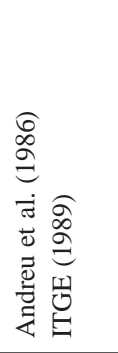 & 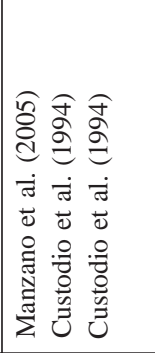 & 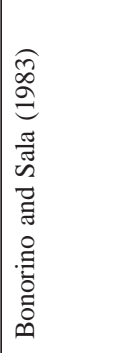 & 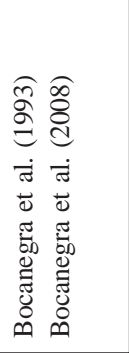 & 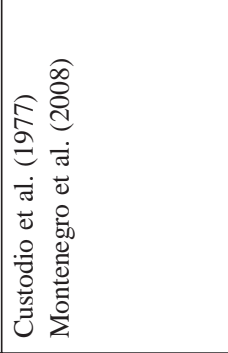 & 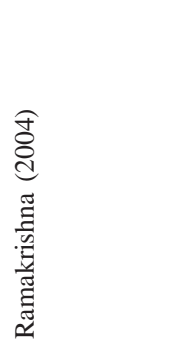 \\
\hline 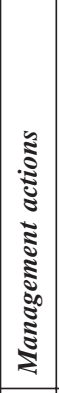 & 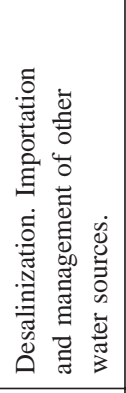 & 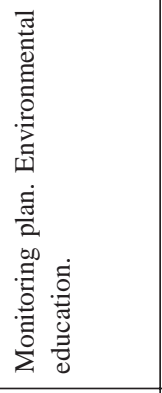 & 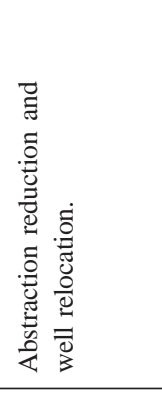 & 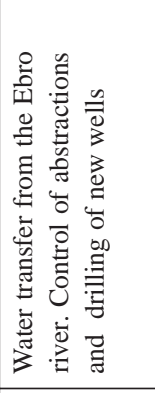 & 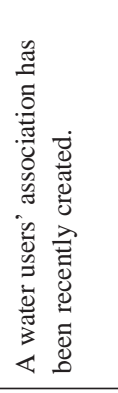 & 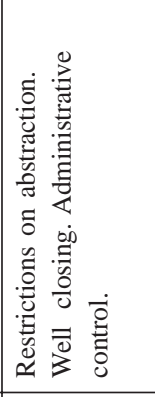 & 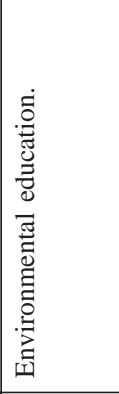 & 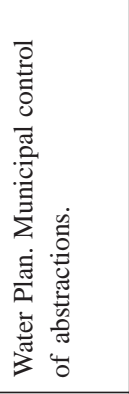 & 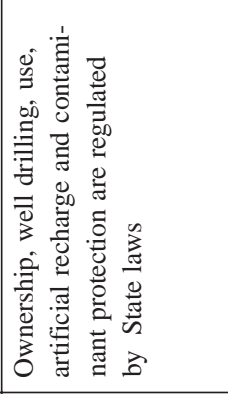 & 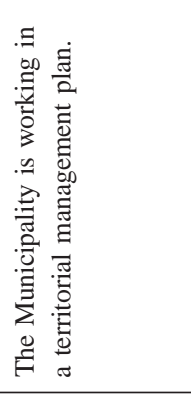 \\
\hline 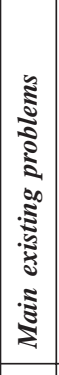 & 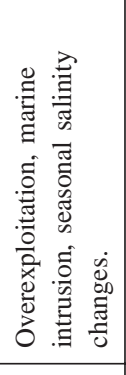 & 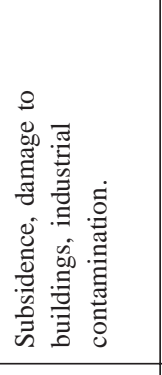 & 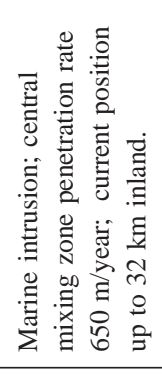 & 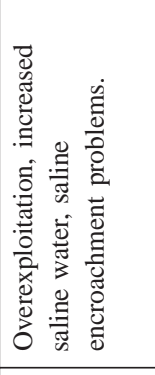 & 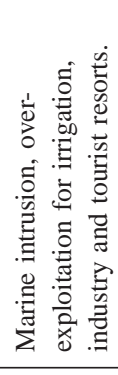 & 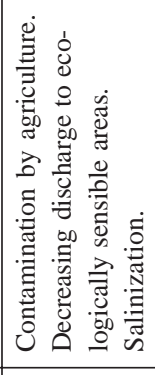 & 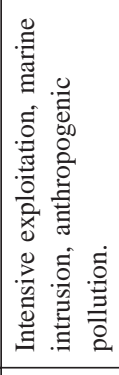 & 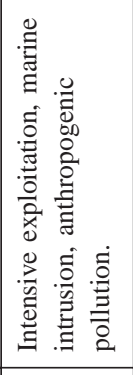 & 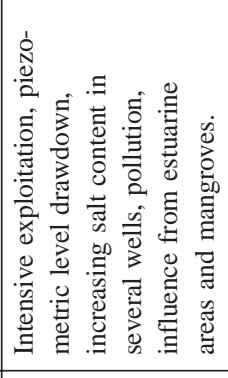 & 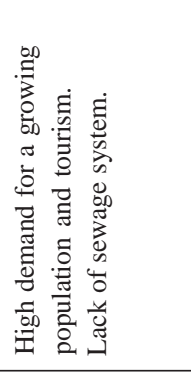 \\
\hline 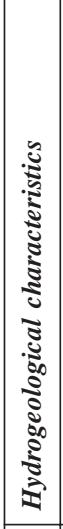 & 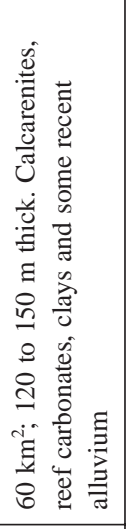 & 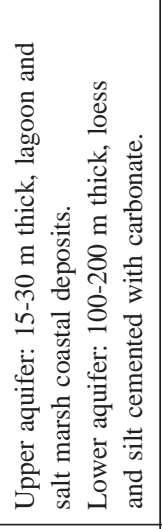 & 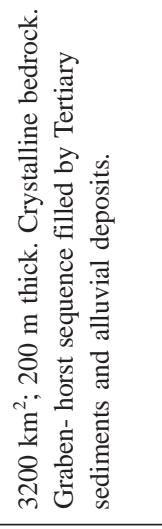 & 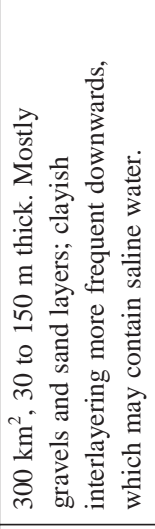 & 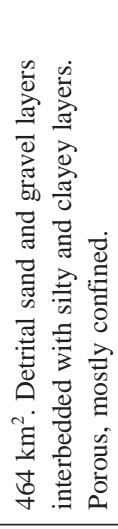 & 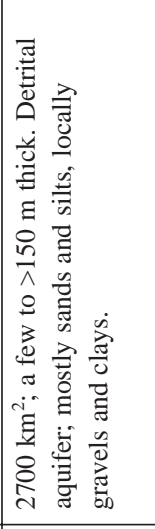 & 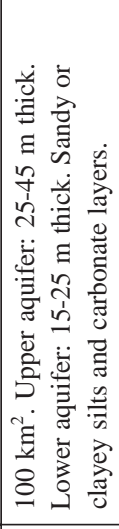 & 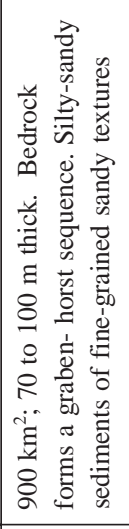 & 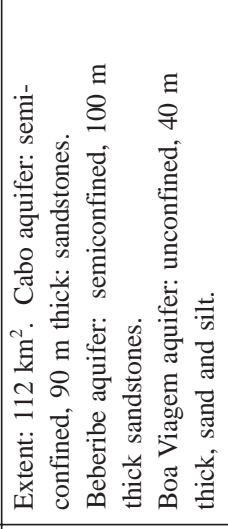 & 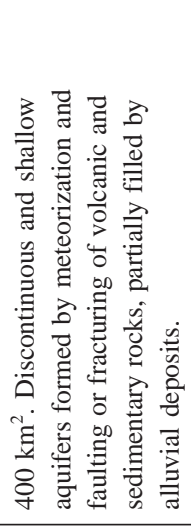 \\
\hline 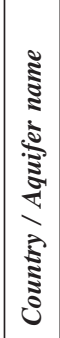 & 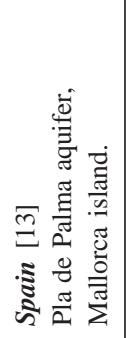 & 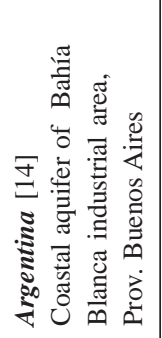 & 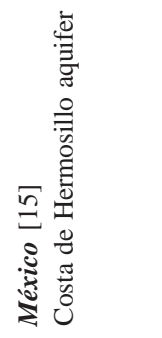 & 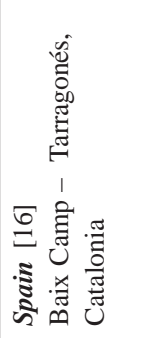 & 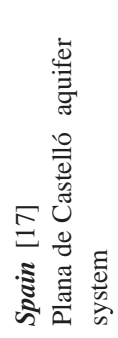 & 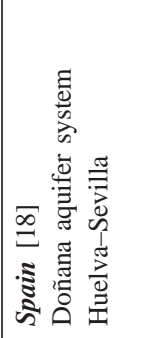 & 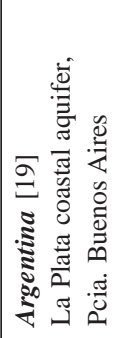 & 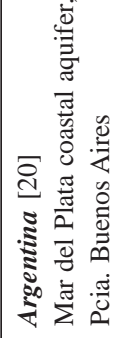 & 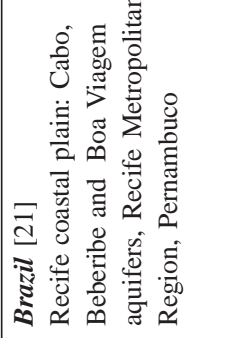 & 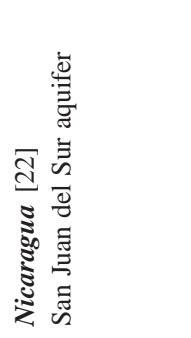 \\
\hline
\end{tabular}




\begin{tabular}{|c|c|c|c|c|c|c|c|c|c|c|c|}
\hline 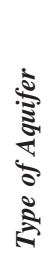 & 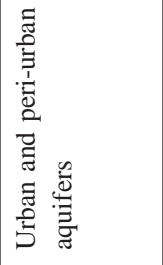 & 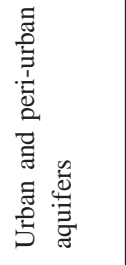 & 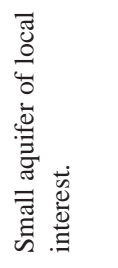 & 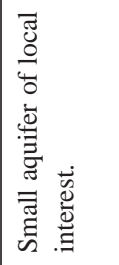 & 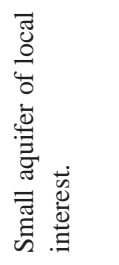 & 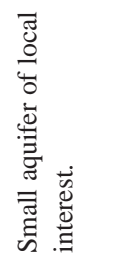 & 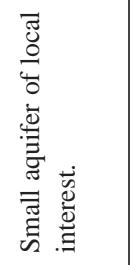 & 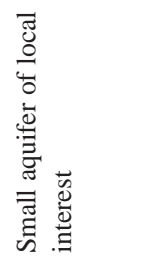 & 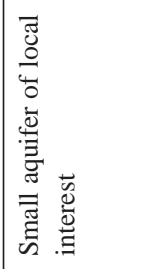 & 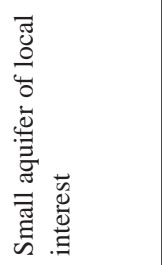 & 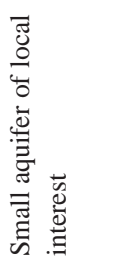 \\
\hline 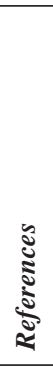 & 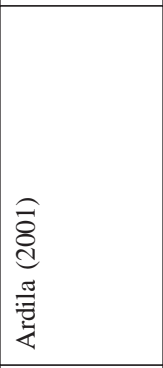 & 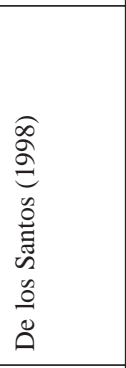 & 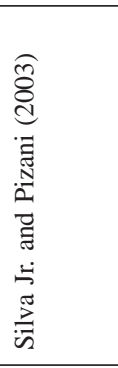 & 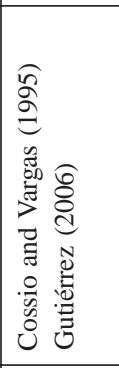 & 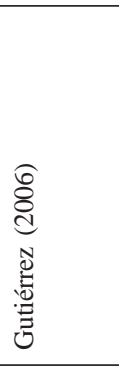 & 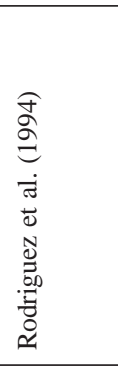 & 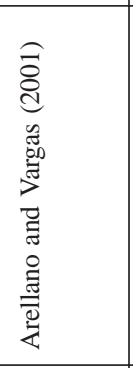 & 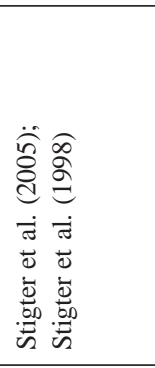 & 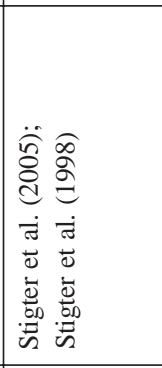 & 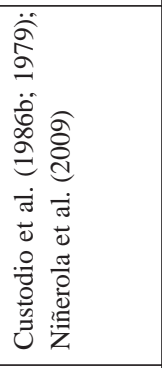 & 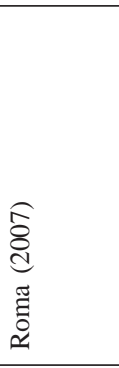 \\
\hline 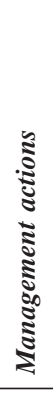 & 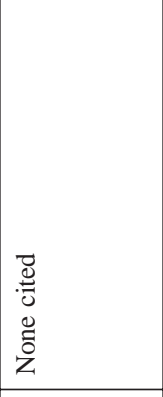 & 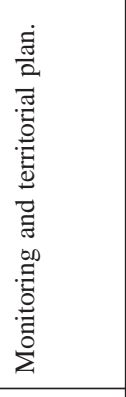 & 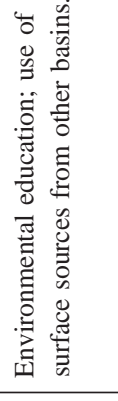 & 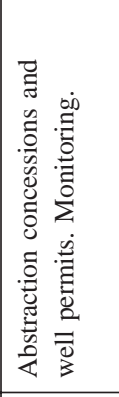 & 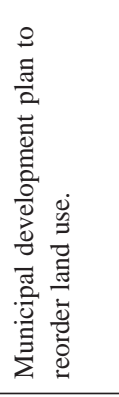 & 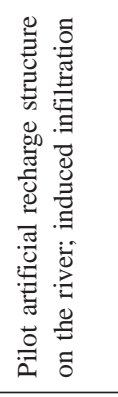 & 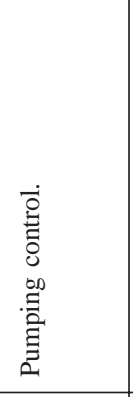 & 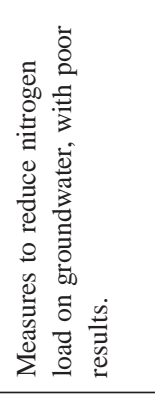 & 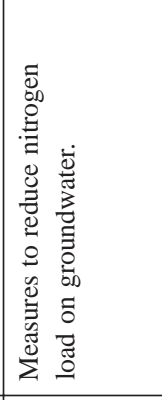 & 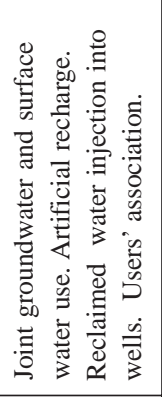 & 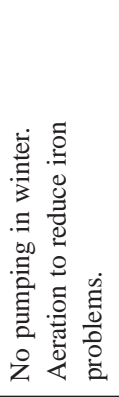 \\
\hline 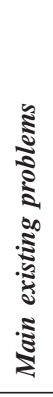 & 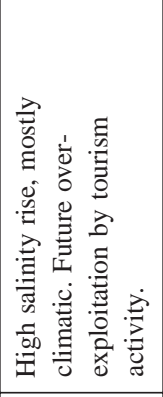 & 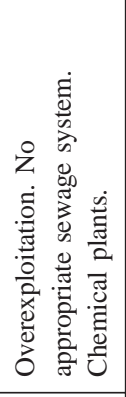 & 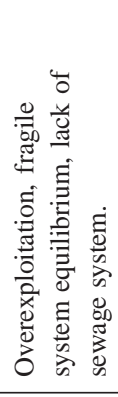 & 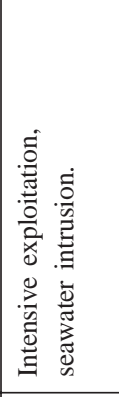 & 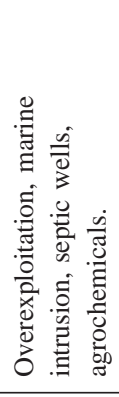 & 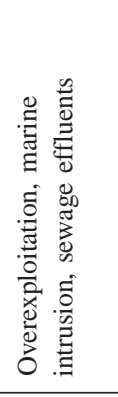 & 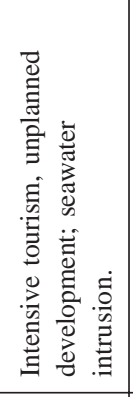 & 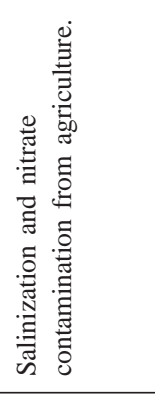 & 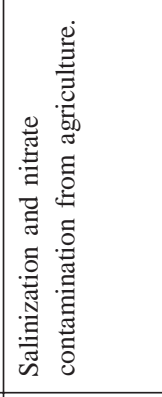 & 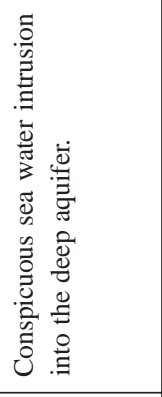 & 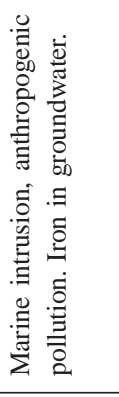 \\
\hline 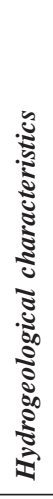 & 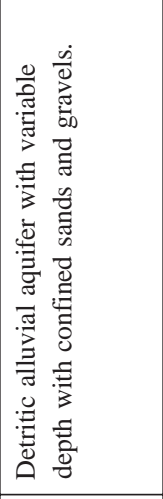 & 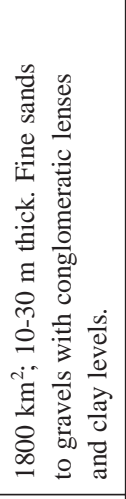 & 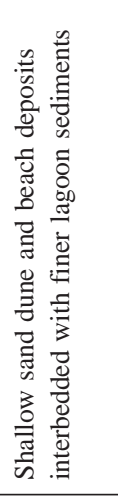 & 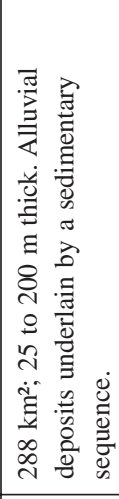 & 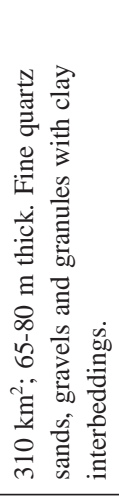 & 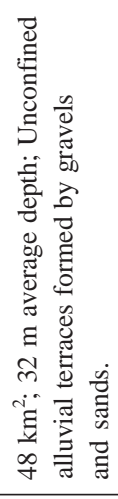 & 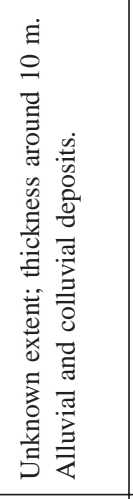 & 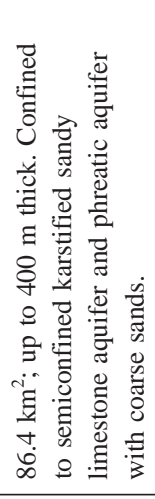 & 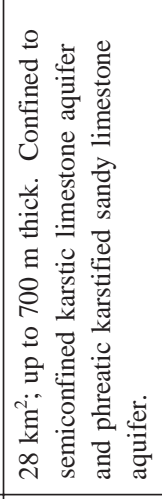 & 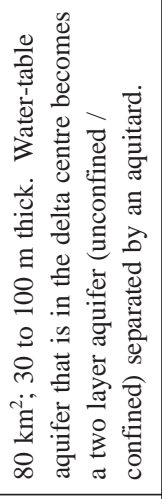 & 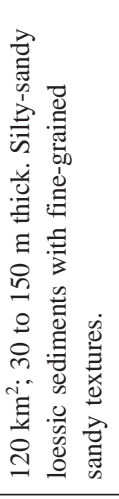 \\
\hline 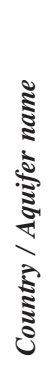 & 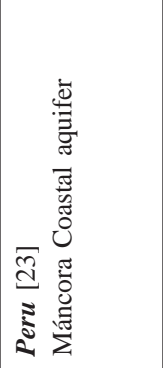 & 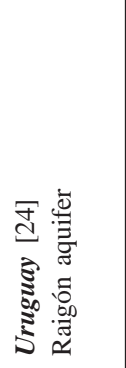 & 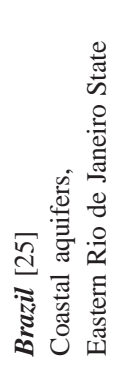 & 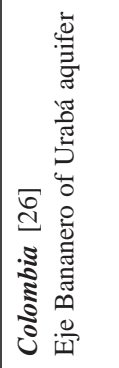 & 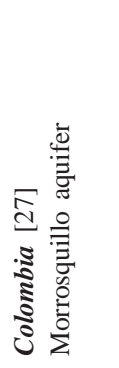 & 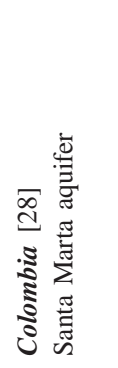 & 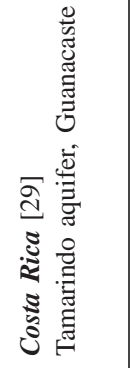 & 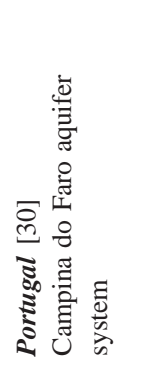 & 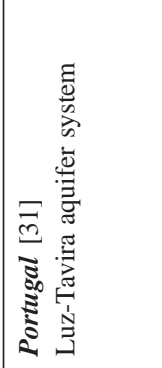 & 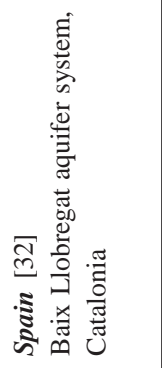 & 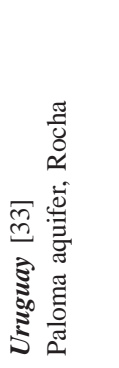 \\
\hline
\end{tabular}


without land and water planning. Decreasing groundwater pumping is advisable, but this must be at least accompanied by other measures, like sewage collection and reuse of treated waste water, as well as relocation of wells.

Carbonated coastal aquifers are prone to increasing permeability due to enhanced calcite dissolution in the mixing zone, leaving cavities. They may be disrupted by pumping and may provoke sinkholes by cave collapsing, which is a serious hazard in urban and peri-urban areas. Also, preferential groundwater flow through underground conduits may facilitate pollutants to unexpectedly reach particular water supply wells or springs, without being noticed by existing monitoring networks.

Thick detrital coastal aquifers are usually highly heterogeneous and anisotropic, which generates relevant spatial permeability variability. Some measures like pumping and new drilling control, or water transfer from foreign basins, have been launched. However, none groundwater and land management plan has been identified.

Mixed coastal aquifers do not show specific situations, but they may experience the problems of different aquifer types at the same time. The studied mixed aquifers share some of the circumstances of carbonated and thick detrital aquifers.

The main common feature for urban and peri-urban coastal aquifers is probably a stressing competence for land and water between urban, industrial and agricultural uses. Regardless of their lithology and dimensions, the local climate, and the existence or not of surface water sources, those aquifers are usually the cheapest water source, so they are heavily exploited and easily contaminated. Salinity, and especially antropic contamination may arise and provoke very serious health problems, most dramatically in some big coastal urban areas in developing countries.

Small coastal aquifers of local interest share many features with urban and peri-urban aquifers, but their dimensions provide some specific factors. Probably the most relevant one is that they act as a water reservoir that may be emptied during the dry season and refilled during the wet one. Thus, groundwater is usually very recent and show quality problems derived from recharge water already impacted by human activities. They show different degrees of knowledge and management, but almost all of them (except the Tamarindo aquifer, in Costa Rica) have been studied for hydrodynamics, groundwater quality and vulnerability.

\section{The Mar del Plata, Recife and Llobregat Delta Case Studies}

The state of knowledge and management of three selected Iberoamerican coastal aquifers with relatively abundant data, Mar del Plata (Argentina), Recife (Brazil), and Llobregat Delta (Spain) ([20], [21] and [32] in Table 1, respectively), are presented with some detail to highlight cases of successful groundwater development, monitoring and remedial measures, as examples of what can be proposed in the future for a sustainable management in other reviewed aquifers.

\section{Mar del Plata}

Mar del Plata, located in Argentine northern coast, is the main tourist centre of the country, with a stable population of 621,000 inhabitants and a seasonal population exceeding 1 million. Water needs are almost exclusively supplied by groundwater sources. The geology consists of a quartzitic Palaeozoic bedrock overlain by a sedimentary cover of Upper Tertiary and Quaternary deposits. Miocene clayeysandy sediments are found at $100 \mathrm{~m}$ depth, covered by Quaternary loess-like sediments. From a hydrogeological viewpoint, Quaternary sediments are the most important reservoir, forming a multilayered phreatic aquifer with thickness ranging from 70 to $100 \mathrm{~m}$, hydraulic conductivity of $10-15 \mathrm{~m} /$ day, and transmissivity of about $600-$ $800 \mathrm{~m}^{2} /$ day in the urban area and between 1000 to $1400 \mathrm{~m}^{2} /$ day in the rural area (Bocanegra et al., 2007). The storage coefficient obtained from pumping tests is 0.001 , and porosity is approximately 0.15 . The average rainfall in 1930-2009 was $910 \mathrm{~mm} /$ year in this city. Natural discharge is towards the sea. Artificial discharge due to pumping has historically lead to significant aquifer head decline.

The most commonly occurring environmental problems related to water resources are: high urban expansion, seawater intrusion, agricultural pollution of groundwater, inadequate waste management, and recurrent or rare flash floods in urban and surrounding areas. Although salinity problems occur, in this case nitrate and biological pollution are more relevant than chloride as pollution indicators.

Regarding management actions, the use of indicators of pressure, state and response to analyse groundwater conditions in Mar del Plata was considered by Bocanegra et al. (2007) as the most effective tool for groundwater management decision making. Despite the critical political and economic context, there have been satisfactory answers to the problems and demands originated around the groundwater resource. Evaluation of global and climate change effects on the role and conditions of Mar del Plata aquifer could be possible with the same indicators used by Bocanegra et al. (2007), that could eventually allow prospective of future behaviour facing those problems.

\section{Recife}

Recife, located in Brazilian northeastern coast, is one of the largest cities in Brazil, with approximately 3.7 million inhabitants in the Metropolitan Area. It is located on a flat lying plain (Recife Plain) ranging from $1 \mathrm{~m}$ to $10 \mathrm{~m}$ above sea level, with the Atlantic Ocean to the East. Geology comprises a basement of crystalline rocks, covered by sedimentary rocks of the Pernambuco-Paraíba basin and volcanosedimentary rocks of the Cabo Basin. The crystalline basement crops out in the western portion of the Metropolitan Area. The Recife Plain aquifer system consists of two semi-confined deep aquifers, Cabo and Beberibe, overlain by a water table aquifer, Boa Viagem. The Cabo aquifer consists of sandstones, siltstones and mudstones, presenting an average thickness of 100 meters. The Beberibe aquifer, to the north of the dominant Pernambuco lineament, has an average thickness of $100 \mathrm{~m}$ and consists of sandstones with interbedded siltstones and mudstones. The Boa Viagem aquifer, with an average thickness of 40 meters, covers the Beberibe and Cabo aquifers, and is composed of sands, silts and clays. In this aquifer, groundwater is easily exploitable and plays a fundamental role in the recharge of the two underlying aquifers. The Beberibe Formation is the most exploited aquifer in this area (Montenegro et al., 2008). The semi-confined Cabo aquifer has the largest number of wells in exploitation, but with relatively low discharge rates, and is an important source of private water supply for Recife. Boa Viagem aquifer has a much higher specific capacity than the other aquifers. Transmissivity and storage are relatively low in all aquifers.

Presently, under intensive exploitation conditions, the aquifers 
are vulnerable to degradation by different causes. Several wells in this region have been showing increasing salt content over the years, and in some cases they were disabled but in others simply abandoned, increasing the risk of aquifer contamination from several sources. The southern area of the Cabo aquifer is intensively exploited, which may explain the salinity increase by seawater intrusion. Salinization also can be caused by wells interconnecting different aquifers, with the upper aquifer contaminated by sewage disposal, presence of mangroves and connection with river estuaries. The vertical flow through the semipermeable layer that connects the two aquifers is another probable cause of the upconing salinization of the Cabo aquifer. Either the way, the condition of intensive exploitation of the aquifer system has a decisive influence on the increasing levels of groundwater salinity in the Plains of Recife.

\section{Llobregat Delta}

The Llobregat River lower valley and delta are located at the southwest of the Barcelona's Metropolitan Area, to the Northeast coast of Spain. They consist of about $30 \mathrm{~km}^{2}$ of alluvial valley up to $1 \mathrm{~km}$ wide, and a delta of roughly $100 \mathrm{~km}^{2}$. The system contains very transmissive aquifers fed by rainfall infiltration, by small creeks draining the mountainous boundary, and by the Llobregat river water infiltration through the river bed and in the irrigated agricultural areas along the valley, which use river water. The aquifer system is a key groundwater source and reserve for a densely populated area (ca. 4 million), besides industrial settlements and remaining irrigated agriculture (Niñerola et al., 2009).

The delta is formed by a sedimentary package ranging from Pliocene to Quaternary in age, the current shape resulting of the delta's progradation during the last Holocene post—glacial marine high stand. In the low valley there is one unconfined aquifer, while in the delta there are one upper and unconfined aquifer and one deep and confined aquifer. These two aquifers partially merge at the delta boundaries. Both aquifers are separated by a wedged body of silt, clays and sandy clays, its thickness increasing towards the sea at the time that produces a more effective isolation of the two superimposed aquifers. The most important aquifer layer for human water supply is the one of the low valley and deep delta's aquifer, this last being also economically important for industries (Niñerola et al., 2009).

Intensive groundwater abstraction produced severe quantity and quality problems already detected more than 30 years ago, which prompted the Water Authority to develop studies during the last four decades. An important landmark for the aquifer management was the creation in 1975 of a Groundwater Users Community aiming to improve the situation and halt the disposal of contaminants in the territory, especially as filling materials of former gravel pits. But the main problem is marine intrusion in the most used deep, confined aquifer. The situation worsened along the years, and it urged to develop more detailed geological and hydrogeological studies ending with groundwater flow and transport modelling. It also prompted the design and implementation of a coastal injection barrier which uses highly treated waste water. Other severe pollution problems, like the appearance of dissolvents in supply wells have been afforded by the own users through the air stripping technique. After the 1985 Spanish Water Act, and especially after the enactment of the European Water Framework Directive of 2000 (WFD), the aquifer status is regularly monitored and deterioration trends are being identified in order to design reversing actions and thus complaint the WFD objective of good chemical and quantitative status (Vázquez-Suñé et al., 2006).

Other management actions under rule are artificial recharge in ponds, induced recharge though river bed scarification, and the combined use of groundwater and surface water (river water and treated water) along the year.

\section{Discussion and Concluding Remarks}

The comparative study performed under the scope of UNESCO IGCP 519 international cooperation project of, regarding 33 coastal aquifers in Iberoamerica, has revealed some common features in terms of groundwater use, problems and groundwater management, such as:

- intensive exploitation;

- need for studies to support planning and management actions at the needed level;

- need for monitoring;

- need for arising awareness and involvement of users and society in management, planning and actions.

Besides, the analysis reinforces that control management actions, beyond economic considerations and specific works, depend upon:

- characteristics of the system;

- participation (society, stakeholders).

International integration could improve promotion of sustainable management in endangered coastal aquifers. An example of international cooperation regarding coastal aquifers in Europe is SALTRANS project (SALTRANS, 2004). The final document stresses that international cooperation and periodic meetings of specialists, such as SWIM meetings, have advanced the state-of-the-art on seawater intrusion.

In general, a strong correlation between economic and human development and aquifer problems awareness and management can be observed. This is clearly seen for the case of urban and peri-urban aquifers, which have the largest number of aquifer studies and of management actions.

The aquifers with fewer management activities are usually those in which there is a low level of knowledge regarding their geometry, flow dynamics and hydrogeology. Conversely, aquifers that are well known, regularly monitored and to which modern and more sophisticated techniques have been applied (such as hydrogeochemical and isotopic techniques, flow and transport modelling, evaluation of marine intrusion and contamination risk assessment) usually have diverse management strategies. Common activities include remediation by artificial recharge, decrease and relocation of extractions, and even procedures to modify the dynamics of saltwaterfreshwater relationships by means of freshwater injection and saltwater abstraction.

In some cases, legal and regulatory activities to protect groundwater resources are scarce or nonexistent, whereas in other cases they constitute the support of an effective management plan. It was noticed that, in general, the creation of effective institutions that reunite and successfully represent the users and the civil society is a necessary step for monitoring and to propose and implement adequate management strategies.

Nonetheless, this approach also requires important efforts to build technical knowledge and credibility. 
The greatest challenge in coastal areas is the lack of awareness of most decision makers and users regarding the negative impact of aquifer overexploitation and contamination. Another important deficiency is the lack of financial resources for scientific and applied research, which are most necessary in order to generate and implement alternatives of sustainable management of coastal aquifers.

International co-operation could help improve the current situation of coastal areas in the Iberoamerican region and thus to promote sustainable management of endangered coastal ecosystems. Efficient water-management policies would help to dramatically reduce the vulnerability of groundwater systems to marine intrusion and impairment, and consequently the present degradation risk for biodiversity, human population and economic development in coastal areas of at least thirty three different systems.

Typification of coastal aquifers based on common scenarios, as presented here for Iberoamerica, could be used in other regions of the world, with the eventual inclusion of other aquifer types that do not exist in the region. A simple and representative characterization of the world's coastal aquifers could be achieved, presenting their state of knowledge, main problems and management actions. On the other hand, comparing the situation of particular aquifers within the same group is very useful to detect the nature of lacks in every area.

The aquifers of Mar del Plata (Argentine), Recife (Brasil) and Llobregat Delta (Spain) are detrital, large size and urban and periurban. The three of them supply large populated areas and also industries and agriculture. All of them have different degree salinization problems, as well as other pollution problems. In the Llobregat Delta, the early awareness of the users impelled the Water Administration to support studies and management actions since late in the 1970 up to nowadays, when a sophisticated set of actions like artificial and induced recharge, injection of treated fresh water in the coast, and planned use of the aquifer as a reservoir, are ruling regularly. In Mar del Plata, detailed studies of the aquifer functioning, including groundwater flow modelling, are under way, and they are used to support the design of the public exploitation network. Also, the use of pressure, state and response indicators to analyse groundwater conditions is supporting management action from the public institutions. In the Recife Plain, salinization and other pollution problems have been detected, but the concrete sources and mechanisms are yet to be identified. A Legal Act from 2000 established criteria for analyzing groundwater requests in intensively exploited regions, but management actions to control groundwater use are still fragile.

\section{Acknowledgements}

The authors wish to thank IGCP 519 UNESCO project for financial support to the working group. The authors also thank the valuable comments and suggestions of two anonymous reviewers.

\section{References}

Andreu, J; Ferrer, J; Estrella, T., 1986, Management of a coastal aquifer with saline intrusion: the case of La Plana de Castellón (Spain): Conjuctive water use, (S.M. Gorelick, Ed.), IAHS Publ. no. 156, pp. 523-34.

Ardila, J.E., 2001, Determination of project storms to Piura Province [in Spanish]: Doctoral Thesis, University of Piura, Perú, pp. 1-112.
Arellano, F., and Vargas, A., 2001, Saline intrusion contamination cases in coastal aquifers of Nicoya Peninsula (Costa Rica) [in Spanish]: Revista Geológica de América Central, v. 25, pp. 77-84.

Barón, A., and González, C., 1995, Salinization of the Llano de Palma aquifers, Mallorca, Spain: Reports by the Junta d'Aigües de Balears, Balearic Islands, Spain. (Only partly published).

Bayó, A., Loaso, C., Aragonés, J.M., and Custodio, E., 1992, Marine intrusion and brackish water in coastal aquifers of Southern Catalonia and Castelló (Spain): a brief survey of actual problems and circumstances: Study and Modelling of Salt Water Intrusion, (E. Custodio, and A. Galofré, Eds.), CIMNE-UPC, Barcelona, pp. 741-766.

Bocanegra, E.M., Martínez, D.E., Massone, H., and Cionchi, J.L., 1993, Exploitation effect and salt water intrusion in the Mar del Plata aquifer, Argentina: Study and Modelling of Salt Water Intrusion into Aquifers, (E. Custodio, and A. Galofré, Eds.), CIMNE-UPC, pp. 177-191.

Bocanegra, E.M., Polemio, M., Massone, H.E., Dragone, V., Limoni, P.P., and Farenga, M., 2007, Indicators and quality classification applied to groundwater management in coastal aquifers: case studies of Mar del Plata (Argentina) and Apulia (Italy): Proceedings of Symposium HS1001 at IUGG2007, Perugia, IAHS Publ. 312, pp. 201-211.

Bocanegra, E.M., Massone, H.E., Cionchi, J.L., Martínez, D.E., 2008, Integrated management of the coastal aquifer in Mar del Plata, Argentina: Proc. 1st SWIM-SWICA Joint Saltwater Intrusion Conference, (G. Barroccu, Ed.), University of Cagliari, pp. 129-134.

Bocanegra, E.M., Custodio, E., Silva Jr, G.C., Manzano, M., and Montenegro, S., 2010, State of knowledge of coastal aquifer management in South America: Hydrogeology Journal, v. 18, pp. 261-267.

Bonorino, A. G., and Sala, J.M., 1983, Geohydrology: Soil Study Comisión White-Cerri, [In Spanish]: Ministerio de Obras Públicas, Provincia de Buenos Aires, La Plata, Final Report (Internal).

Bonorino, G.A., and Albouy, E.R., 2003, Hidrodinámica del área costera de Ingeniero White, [In Spanish]: Final Report, Comité Técnico Ejecutivo, Secretaría de Política Urbano Ambiental, Municipalidad de Bahía Blanca, pp. 1-22.

Cabrera, M.C., and Custodio, E., 2004, Groundwater flow in a volcanicsedimentary coastal aquifer: Telde area, Gran Canaria, Canary Islands, Spain: Hydrogeology Journal, v. 12, no. 3, pp. 305-320.

Cossio Ubaldo, M.C., and Vargas, A., 1995, Groundwater evaluation in Urabá Region, [In Spanish]: Final Report, Instituto de Investigaciones en Geociencias, Minería y Química, CORPOURABA, Bogotá, Colombia (Internal).

Custodio, E., 1979, Artificial recharge in the coastal aquifers near Barcelona (Spain), Selected Water Problems in Islands and Coastal Áreas with special regards to Desalinization and Groundwater: Pergamon Press, pp. 465-472.

Custodio, E. and Bruggeman, G.A., 1987, Groundwater problems in coastal areas. Studies \& Reports in Hydrology. no. 45, UNESCO, Paris, pp. 1576.

Custodio, J., and Custodio, E., 2001, Isotopic hydrogeochemistry in Amurga phonolitic massif (SE of Isla de Gran Canaria), [In Spanish]: Las Caras del Agua Subterránea (A. Medina and J. Carrera, Eds.), Barcelona, IGME. Madrid, v. 1, pp. 461-468.

Custodio, E., Cruz, W., Braz da Silva, A., Jardim, F., and Peixoto, C.A., 1977, Estudo sobre as possibilidades de intrusão marinha no aqǘf́ro Beberibe, Região Costeira de Olinda-Goiana (PE): Rev. Geociências, Soc. Brás. Geologia. São Paulo, v. 7, no. 3, pp. 239-255.

Custodio E, Pascual, J.M., Bosch, X., and Bayó, A., 1986a, Sea Water intrusion in coastal carbonate formations in Catalonia, Spain: Proc. 9th Salt Water Intrusion Meeting, Delft University of Technology, pp. 147-164.

Custodio, E., Iribar, V., Manzano, M., Bayó, A., and Galofré, A., 1986b, Evolution of sea water intrusion in the Llobregat delta, Barcelona, Spain: Proc. 9th Salt Water Intrusion Meeting, Delft University of Technology, pp. 133-146.

Custodio, E., Barón, A., Rodríguez Morillo, H., Poncela, R., and Bayó, A., 1992, Saline water in S'Albufera Natural Park aquifer system, Mallorca Island (Spain): A preliminary study: Study and Modelling of Salt Water 
Intrusion, (E. Custodio, and A. Galofré, Eds.), CIMNE-UPC, Barcelona, pp. 661-686.

Custodio, E., Iglesias, M., Manzano, M., and Trick, T., 1994, Saltwater intrusion along the western Doñana area coast (Southwestern Spain): Proc. 13th Salt Water Intrusion Meeting, G. Barrocu (Ed.), pp. 287-303.

De los Santos, J., 1998, Numerical Modeling of a bilayered aquifer. Calibration in steady state (part 1), [in Spanish]: XVIII Congreso Latinoamericano de Hidráulica, Oaxaca, México.

Domínguez, P., and Custodio, E., 1992, Seawater intrusion in the lower northeastern aquifer of the "Campo de Dalias" (Almería, Southeastern Spain): preliminary study of monitoring data: Study and Modelling of Salt Water Intrusion, (E. Custodio, and A. Galofré, Eds.), CIMNE-UPC, Barcelona, pp. 631-659.

Gutiérrez, GE., 2006, Diagnóstico Ambiental del Acuífero Costero Golfo de Morrosquillo, Zona Litoral Tolú-Coveñas, Power Point presentation [in Spanish]: $14^{\circ}$ Reunión Asociación Avances para la Ciencia, CARSUCRE, Colombia.

Herrera, Ch., and Custodio, E., 2000, Saline water in Central Fuerteventura Island, Canary Islands, Spain: Proc. $16^{\text {th }}$ Salt Water Intrusion Meeting, Miedzyzdroje-Wollin Island, Poland, v. 80, pp. 81-86.

Herrera, Ch., and Custodio, E., 2008, Conceptual hydrogeological model of volcanic Easter Island (Chile) after chemical and isotopic surveys: Hydrogeology Journal, v. 16, no. 7, pp. 1329-1348.

Herrera, Ch., Pincheira, M., Custodio, E., Araguás, L., and Velasco, G., 2004, Tritium content in groundwater from Easter Island, Chile, as a tool to assess recharge on a volcanic aquifer, [in Spanish]: Boletín Geológico y Minero, v. 15, pp. 299-310.

INGEOMINAS, 1993, San Andrés aquifer study and well field recuperation, [In Spanish]: Informe prospección Geoeléctrica en la Isla de San Andres, INGEOMINAS, Bogotá, Colombia, (Internal).

ITGE, 1989, Planas de Castellón and Sagunto, [in Spanish]: Instituto Tecnológico Geominero de España, Serie: Manuales de Utilización de Acuíferos, Madrid.

López Infante, E., 2000, Study of sources to dessalinization plants in Cayo Largo del Sur [in Spanish]. Revista Ingenieria Hidráulica y Ambiental. CIH. Vol. XXII, pp. 39-46.

Manzano, M., Custodio, E., Loosli, H., Cabrera, M.C., Riera, X., and Custodio, J., 2001, Palaeowater in coastal aquifers of Spain, in: W.M. Edmunds, and C.J. Milne, (Eds.), Palaeowaters in Coastal Europe: Evolution of Groundwater since the late Pleistocene. Geological Society London, Special Publication, no. 189, London, pp. 107-138.

Manzano, M., Custodio, E., Mediavilla, C., and Montes, C., 2005, Effects of localised intensive aquifer exploitation on the Doñana wetlands (SW Spain), in: A. Sahuquillo, J. Capilla, L. Martínez-Cortina, and X. Sánchez Vila, (Eds.), Groundwater intensive use. IAH Selected Papers on Hydrogeology 7, Balkema, pp. 209-219.

Marín, L., 1990, Field investigation and numerical simulation of ground water flow in the karstic aquifer of northwestern Yucatán, Mexico: PhD Thesis, Northern Illinois University, Dekalb, Illinois, USA.

Marques da Silva, M.A., 1988, Parámetros geohidrológicos y geohidroquímicos de los niveles permeables profundos y semiprofundos del área de la Ria de Aveiro (Portugal): Doctoral Thesis, Technical University of Catalonia, Barcelona (Spain) and University of Aveiro (Portugal).

Melo, M.T.C., Marques da Silva, M.A., and Edmunds, W.M., 1998, Evolução Hidrogeoquímica do Sistema Multiaquífero Cretácico do Baixo Vouga Aveiro, Portugal, [In Portuguese]: Proc. 4th Congresso da Água, APRH.
Molina, L., Pulido-Bosch, A., Vallejos, A., Calaforra, J.M., Navarrete, F., and Martín Rosales, W., 2000, Hydrogeological problems in Campo de Dalías (Almería), [In Spanish], Proc. Iberian Congress on Water Planification and Management, Zaragoza, 1998, in CD.

Montenegro, S.M.G.L., Montenegro, A.A. de A, Cabral, J.J.S.P., and Cavalcanti, G., 2008, Intensive exploitation and groundwater salinity in Recife coastal plain (Brasil): monitoring and management perspectives: Proc. Proc. 1st SWIM-SWICA Joint Saltwater Intrusion Conference, G. Barroccu, (Ed.), University of Cagliari, pp. 79-85.

Niñerola, J.M., Queralt, E., and Custodio, E., 2009, Llobregat Delta Aquifer, in: Groundwater Monitoring, Ph. Quevauviller, A. M. Fouillac, J. Grath, and R. Ward (Eds.), John Wiley \& Sons, pp. 289-301.

Ramakrishna, B. (Coord), 2004, Gestión Integrada de los Recursos Hídricos de la Cuenca del Río San Juan y su Zona Costera Mar, [In Spanish]: Org. American States, 3 Oct. 2008. in: <http://www.oas.org/sanjuan/spanish/ documentos/adt/informacion/sobreexplotacion.html>

Rangel Medina, M., Monreal, R., Morales, M., and Castillo Gurrola, J., 2004, Estimation of the vulnerability to saline intrusion of the coast of Hermosillo aquifer, Sonora, Mexico: Geofísica Internacional, v. 43, no. 4, pp. 611-621.

Rodríguez, C.O., Molano, C., Bonilla, F., 1994, Artificial recharge of the Santa Marta Aquifer, Colombia: Artificial Recharge of Ground Water, II Symposium, New York, v. II, pp. 446-455.

Rodríguez Estrada, H., 2002, Groundwater as an alternative source to Cahuita, Limón, [In Spanish], in: J. Reynolds-Vargas (Ed.), Manejo Integrado de Aguas Subterráneas. Editorial EUNED, Costa Rica, v. 1, pp. 143-152.

Roma, M.T., 2007, Informes sobre el acuífero de La Paloma, (In Spanish): Report for Obras Sanitarias del Estado (OSE), Montevideo, (Internal).

Rudovol, A., 1974, Hydrogeological study of Cuenca Sur de La Habana, [In Spanish]: Informe Técnico, Instituto Nacional de Recursos Hidráulicos, La Habana, Cuba, (Internal).

SALTRANS, 2004, Methods for assessing salt intrusion and transport in heterogeneous and fractured aquifers: Basic principles and concepts for understanding and managing sea water intrusion in coastal aquifers, European Commission Programme: Energy, Environment and Sustainable Development, Final Report, pp. 1-24.

Schneider, E.G., 2003, Estado actual y evolución de la intrusión marina en los acuíferos costeros del litoral meridional de Tarragona (España), [In Spanish]: Tecnología de la intrusión marina en acuíferos costeros: países mediterráneos, IGMe, Madrid, Spain, pp. 29-38.

Silva, Jr. G.C., and Pizani, T.C., 2003, Vulnerability Assessment in Coastal Aquifers Between Niterói And Rio das Ostras, Rio de Janeiro State, Brazil: Rev. Latinoamericana de Hidrogeología, v. 3, no. 2, pp. 125-133.

Stigter, T.T., van Ooijen, S.P.J., Post, V.E.A., Appelo, C.A.J., and Carvalho Hill, A.M.M., 1998, A hydrogeological and hydrochemical explanation of the groundwater composition under irrigated land in a Mediterranean environment, Algarve, Portugal: Journal of Hydrology, v. 208, 262-279.

Stigter, T.T., Almeida, P., Carvalho Dill, A.M.M., and Ribeiro, L., 2005, Influence of irrigation on groundwater nitrate concentrations in areas considered to have low vulnerability to contamination, in: E.M. Bocanegra, M.A. Hernández, and E. Usunoff, (Eds.), Groundwater and Human Development. Selected Papers on Hydrogeology 6, Balkema, Leiden, Holanda, pp. 69-85.

Vázquez-Suñé, E., Abarca, E., Carrera, J., Capino, B., Gámez, D., Pool, M., Simó, T., Battle, F., Niñerola, J.M., and Ibáñez, X., 2006, Groundwater modelling as a tool for the European Water Framework Directive (WFD) application: The Llobregat case. Physics and Chemistry of the Earth, v. 31, no. 1, pp. 1015-1029. 


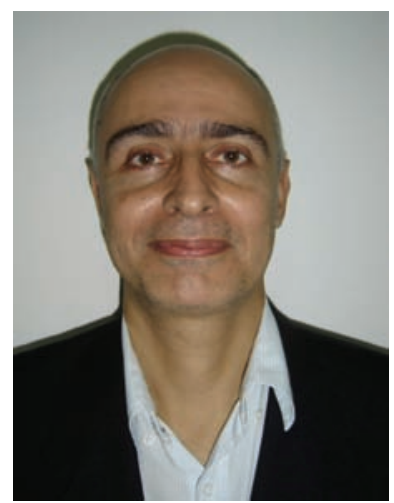

Gerson Cardoso da Silva Jr. leads the Hydrogeology Research Group at the Federal University of Rio de Janeiro, Brazil (UFRJ), supported by the Brazilian National Research Council and other Institutions. Silva Jr. received a Ph.D. in Geology (Hydrogeology) from Polytechnic University of Catalonia, Spain, in 1997. His present research is focused on comprehension of coastal aquifer functioning mechanisms through hydrodynamic studies, isotope and geochemical tools.

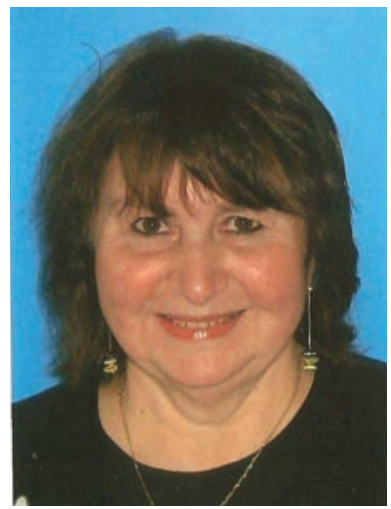

Emilia Bocanegra, Professor and Chairperson of the research Hydrogeology Group at the National University of Mar del Plata, Argentina She has written more than 75 papers. She is leader of national Projects on assessment of groundwater resources and leader of both UNESCO and IAEA projects on integrated management of coastal aquifers in Iberian America.

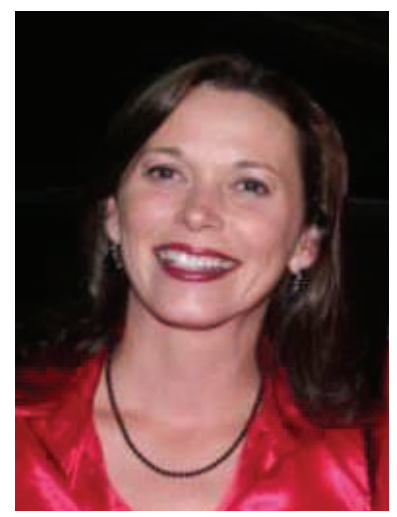

Suzana Maria Gico Lima Montenegro, Associated Lecturer in Hydrology and Water Resources at Civil Engineering Department, in the Federal University of Pernambuco, Brazil (UFPE). She received $\mathrm{Ph} . \mathrm{D}$. in Water Resources from University of Newcastle Upon Tyne, England, in 1997. Her present research is focused on comprehension of coastal aquifer functioning mechanisms through hydrodynamic and hydro chemical studies and modeling, and groundwater management in semiarid regions.

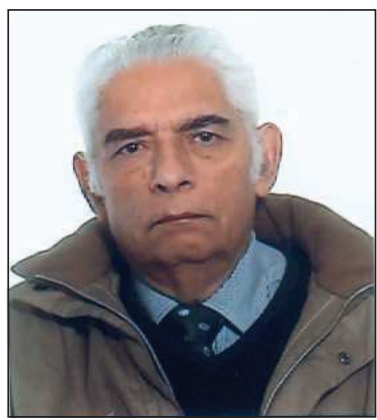

Emilo Custodio, Full Professor of Geo-engineering (Groundwater Hydrology) in the Civil Engineering School of the Technical University of Catalonia, Barcelona, Spain. His main focus is on groundwater hydrology and water resources, with emphasis on general evaluations, hydrogeochemistry, environmental isotope techniques, aquifer recharge, coastal aquifers, volcanic islands hydrogeology and groundwaterdependent wetlands.

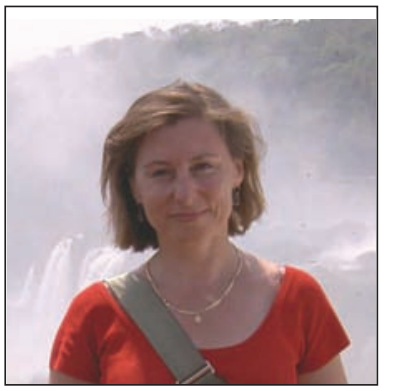

Marisol Manzano, Ph.D in Geology in 1993 from the Technical University of Catalonia, Spain. Since 2001 she is a Professor in groundwater hydrology at the Technical University of Cartagena, Spain. Her current research projects are in Spain, Argentina and Uruguay, dealing with the use of specific tracers to understand groundwater-surface waters relationships in big wetland areas. 